\section{Mehr Lebensqualität}

Brustkrebspatientinnen nach brusterhaltender Op schätzen ihre Lebensqualität umso höher ein, je zufriedener sie mit dem ästhetischen Ergebnis sind. Das hat eine Studie mit rund 1000 Frauen belegt. Per Fragebogen und Software lässt sich das subjektive Behandlungsergebnis systematisch erfassen. So könnten künftig brusterhaltende Therapiekonzepte in Bezug auf Ästhetik und Lebensqualität verglichen werden.

Ärzte Zeitung, 2.2.2013

\section{Tiefer Einblick}

Ein detaillierter Blick unter die Haut, der Krebs-Vorstufen bzw. den Verlauf von Hautkrebserkrankungen erkennen lässt - eine neue Lasertechnologie macht dies nach Angaben einer Pressemeldung der Medical University of Vienna möglich. So gelingt eine detailreiche Darstellung der Durchblutung und der Gefäßstruktur. „Damit ist es erstmalig gelungen, derartig detaillierte Bilder des Gefäßsystems von Hautkarzinomen beim Menschen zu bekommen“, so ein Experte.

hautnah dermatologie 2013, 29 (1):7

\section{Schlappes Essen, schlappe Haut}

Die Ernährung scheint bei der Entwicklung der lichtabhängigen Hautalterung und Elastose eine Rolle zu spielen. Nach einer Untersuchung mit 136 Patienten, die sich wegen eines Basalioms im Gesicht einer Operation unterzogen, besteht eine positive Assoziation einer Vitamin-E- und -C-reichen Ernährung mit viel Obst und Tee und einer geringeren Ausprägung der Elastose.

Husein-EIAhmed $\mathrm{H}$ et al, JEADV 2013, 27:25

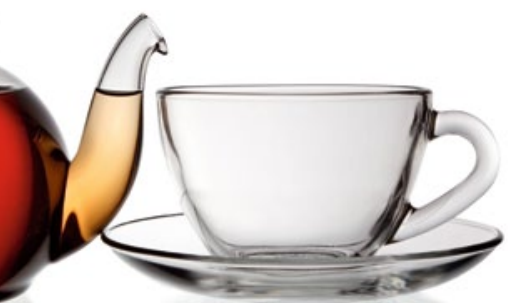

4 ästhetische dermatologie $01 \cdot 2013$

Riskante TV-Vorlieben

\title{
Nach der Beauty-Show auf die Sonnenbank
}

Wer Beauty-Shows im Fernsehen ansieht, neigt offenbar eher dazu, sich unter die UV-Lampe zu legen oder regelmäßig unter freiem Himmel zu brutzeln, als Personen, die andere Fernsehsendungen bevorzugen oder sich lieber gleich mit sinnvolleren Dingen beschäftigen. Dies zumindest ergab eine Befragung amerikanischer College-Studenten.

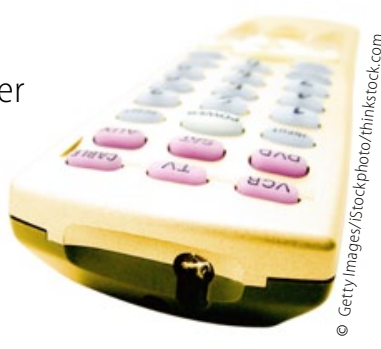

Um herauszufinden, wie stark RealityShows das Verhalten der Zuschauer beeinflussen, haben Wissenschaftler des Brooklyn College in New York 576 Studenten anonym befragt. Die Dermatologen wollten wissen, ob der perfekte Model-Körper bei jungen Leuten zwischen 18 und 29 Jahren Begehrlichkeiten nach mehr brauner Haut weckt. Tatsächlich zeigte sich zwischen denen, die sich die Shows ansahen, und solchen, die dies nicht taten ein deutlicher Unterschied hinsichtlich des Bräunungsverhaltens. 12,9\% der Beauty-ShowFans hatten sich im vergangenen Jahr ins Sonnenstudio gelegt, $43,3 \%$ von ihnen hatten sich länger als zwei Stunden am Stück im Freien gesonnt. Von den Befragten dagegen, die angaben, keine solchen Sendungen zu sehen, nutzten nur 3,7\% künstliche
UV-Quellen und 28,7\% legten sich stundenlang in die pralle Sonne. In der multivariaten Analyse blieb der signifikante Zusammenhang zwischen der Vorliebe für Beauty-Shows und brauner Haut erhalten. Studenten, die sich die Fernsehsendungen ansahen, waren etwa doppelt so häufig in Sonnenstudios und beim Sonnenbad unter freiem Himmel anzutreffen. Dabei wurden künstliche UV-Quellen von älteren weiblichen Studenten am häufigsten genutzt. Dem Einfluss solcher TV-Sendungen müsste nach Ansicht der Autoren aktiv begegnet werden, indem in den Medien auch die Risiken der UV-Bestrahlung für die Haut mehr thematisiert würden.

(st)

Fogel J et al, J AM ACAD DERMATOL 2012 (online first)

\section{Botulinumtoxin gegen fettige Haut}

\section{Botulinumtoxin kann nicht nur Mimikfalten, sondern auch Fett- glanz von der Stirn beseitigen.}

Bisher ist eine Behandlung mit Isotretinoin die wirksamste Maßnahme bei übermäßiger Sebumproduktion. Ähnlich effektiv, aber besser tolerabel könnten nach Einschätzung von Dermatologen der New York University School of Medicine intradermale Injektionen von Botulinumtoxin sein. Die US-Ärzte haben die neue Methode in einer offenen Studie bei 20 Frauen und fünf Männern mit fettender Haut im Stirnbereich ausprobiert. Alle Patienten erhielten einmalig 30-45 Einheiten Abobotulinumtoxin A, verteilt auf zehn Stellen in der Stirnregion. Die Talgproduktion vor und nach der Behandlung wurde mit einem Sebometer sowie anhand von Fotografien und der Patientenzufriedenheit be- urteilt. Das Ergebnis: Zu allen Zeitpunkten nach der Injektion waren die Talgdrüsen weniger aktiv als zuvor. Im Durchschnitt war die Sebumproduktion nach einer Woche um 75\%, nach einem Monat um 80\% und nach zwei bzw. drei Monaten um 73\% bzw. 59\% gedrosselt. Bei allen bis auf einen Patienten wurde eine mindestens 50\%ige Abnahme des Sebums gemessen. 23 Patienten gaben an, mit der Behandlung „zufrieden“ zu sein, einer war sogar ,sehr zufrieden“ und ein weiterer „einigermaßen zufrieden“. „Intradermal injiziertes Botulinumtoxin könnte eine wirksame Behandlung für fettige Haut darstellen und möglicherweise auch bei vergrößerten Poren helfen", lautet das Fazit der Studienautoren um Dr. Amy E. Rose.

(bs)

Rose AE et al, Dermatologie Surgery 2013 (online first) 